\title{
Review Article \\ Looking beyond Androgen Receptor Signaling in the Treatment of Advanced Prostate Cancer
}

\author{
Benjamin Sunkel ${ }^{1,2}$ and Qianben Wang ${ }^{1,2}$ \\ ${ }^{1}$ Department of Molecular and Cellular Biochemistry and the Comprehensive Cancer Center, \\ The Ohio State University College of Medicine, Columbus, OH 43210, USA \\ ${ }^{2}$ Ohio State Biochemistry Graduate Program, The Ohio State University, Columbus, OH 43210, USA \\ Correspondence should be addressed to Qianben Wang; qianben.wang@osumc.edu
}

Received 19 January 2014; Accepted 17 March 2014; Published 10 April 2014

Academic Editor: Wen-Chin Huang

Copyright (C) 2014 B. Sunkel and Q. Wang. This is an open access article distributed under the Creative Commons Attribution License, which permits unrestricted use, distribution, and reproduction in any medium, provided the original work is properly cited.

\begin{abstract}
This review will provide a description of recent efforts in our laboratory contributing to a general goal of identifying critical determinants of prostate cancer growth in both androgen-dependent and -independent contexts. Important outcomes to date have indicated that the sustained activation of AR transcriptional activity in castration-resistant prostate cancer (CRPC) cells results in a gene expression profile separate from the androgen-responsive profile of androgen-dependent prostate cancer (ADPC) cells. Contributing to this reprogramming is enhanced FoxA1 recruitment of AR to G2/M phase target gene loci and the enhanced chromatin looping of CRPC-specific gene regulatory elements facilitated by PI3K/Akt-phosphorylated MED1. We have also observed a role for FoxA1 beyond AR signaling in driving G1/S phase cell cycle progression that relies on interactions with novel collaborators MYBL2 and CREB1. Finally, we describe an in-depth mechanism of GATA2-mediated androgen-responsive gene expression in both ADPC and CRPC cells. Altogether these efforts provide evidence to support the development of novel prostate cancer therapeutics that address downstream targets of AR activity as well as AR-independent drivers of disease-relevant transcription programs.
\end{abstract}

\section{Introduction}

The androgen receptor (AR), a member of the steroid receptor superfamily [1], is a classic example of a ligand-inducible transcription factor whose activity is tightly linked to numerous physiological processes and disease states. Within the various mammalian tissues expressing $A R$, its essential role in organ development and function has been demonstrated, which ranges from contributions to spermatogenesis, sexual behavior, and skeletal maintenance [2]. Mechanistically, induction of AR activity relies upon binding to male hormones, androgens (e.g., testosterone or the more potent $5 \alpha$ dihydrotestosterone [DHT]), resulting in release of AR from stabilizing interactions with cytoplasmic heat shock proteins (HSPs) [3]. Homodimerization, activation by posttranslational modification, and nuclear translocation of ligandbound AR is then essential for its established role in determining tissue-, cell type-, and disease stage-specific gene expression patterns [3]. Within the nucleus, AR binds genomic regions enriched with its cognate DNA-binding motif, or androgen response element (ARE), consisting classically of the 15-base pair sequence $5^{\prime}$-AGAACAnnnTGTTCT- $3^{\prime}$ and providing a degree of specificity to global AR distribution within regulatory elements of androgen responsive genes [4]. These regulatory regions further serve as a substrate for the assembly of AR coactivator and transcription complexes that initiate gene expression.

Advances in the understanding of this AR activity have had no greater impact than in the human prostate cancer field. Development and normal function of the prostate secretory epithelium are understood to rely on hormone-inducible gene expression mediated by AR [2,5], and knowledge of the subsequent dependence on androgens for the malignant proliferation of prostate adenocarcinoma dates back to the Nobel Prize-winning insights of Dr. Charles Huggins, who demonstrated that surgical castration or application of endogenous estrogens resulted in decreased cellular proliferation in benign prostatic hyperplasia $(\mathrm{BPH})$ and regression of local and metastatic prostate cancer [6-9]. Leveraging the sensitivity of prostate cancer to castrate levels 
of androgens, therapeutics targeting testicular and adrenal androgen synthesis (luteinizing hormone-releasing hormone agonists/antagonists [e.g., leuprorelide and cetrorelix, respectively] and steroidogenic cytochrome P450 [CYP] enzyme inhibitors [e.g., ketoconazole]) would later underlie the medical treatment of advanced disease $[10,11]$. Discovery and characterization of AR [12-14] also allowed for the development of nonsteroidal antiandrogens (e.g., flutamide) that could compete with DHT for AR binding, thus inhibiting its transcriptional activity [15].

Today, combination therapy involving androgen ablation and the application of antiandrogens is the mainstay of treatment for metastatic disease and is initially effective against androgen-dependent prostate cancer (ADPC) cases naive to hormone therapy [16-18]. Despite the early response, the heterogeneous nature of prostate cancer cell populations, characterized by both androgen-dependent and -independent sub-populations, provides an initial rationale for the nearly inevitable progression of the disease to a treatmentinsensitive, castration-resistant prostate cancer (CRPC) phenotype. However, recent insights suggest that it is overly simplistic to regard such lethal cases as entirely independent of the activities of AR. As previously reviewed [19-21], a number of mechanisms have been explored that support reestablished AR activity as a prominent driver of CRPC growth even in the presence of the modern, more potent second generation antiandrogen (enzalutamide), and CYP inhibitor (abiraterone) [22-28]. Briefly, through amplification, overexpression, or perhaps stabilization of AR, cancer cells may exhibit enhanced sensitivity to low levels of circulating androgens $[29,30]$. Mutations within the AR ligand binding domain (LBD) or alternative splicing that excludes the LBD altogether can allow cells to utilize antiandrogens as ligands or deviate completely from the need for ligand induction for AR transactivation, respectively [31-36]. Alternately, intratumoral androgen synthesis, via upregulation of steroidogenic enzymes [37-39] or aberrant activation of AR by upstream kinases responding to growth factor stimulation, such as epidermal growth factor (EGF) and insulin-like growth factor 1 (IGF1), may stimulate AR activity following androgen ablation $[40,41]$. Finally, and most relevant to the current review, overexpression or activation of AR coactivators/collaborating transcription factors and lost activity of AR corepressors may contribute to the redistribution of $\mathrm{AR}$, promoting a diseasestage specific transcription profile, or to the activation of AR by antiandrogens [42-44].

It has been demonstrated that the selective pressure applied by the therapeutic strategies described above can result in the outgrowth of treatment-insensitive cancer cell populations utilizing the aforementioned resistance mechanisms [45]. Still other CRPC cells lose expression of AR and are thus entirely refractory to compounds targeting the androgen signaling axis. Our current inability to address the molecular determinants of CRPC growth contributes to the role of prostate cancer as the second leading cause of cancerrelated deaths in American men and encourages continued investigation into the role of $\mathrm{AR}$ as well as additional factors throughout disease progression [46]. The primary focus of our recent investigations, outlined in this review, has been to evaluate alterations to AR-mediated gene expression profiles in the progression from ADPC to CRPC. The elucidation of factors contributing to disease stage-specific redistribution of AR binding has been central to this aim. Emerging from the earliest efforts of our lab, this review will highlight the central importance of the pioneer transcription factors GATA2 and FoxA1 in models of early and late stage disease by describing the manner in which FoxA1 directs CRPC-specific AR binding and drives androgen-independent cell cycle progression and the in-depth mechanism by which GATA2 contributes to androgen/AR-mediated gene expression in both ADPC and CRPC. Finally, in addition to highlighting how the coordinated activities of these factors with the Mediator coregulatory complex subunit MED1 has been shown to facilitate chromatin loop formation/maintenance allowing for enhancer-driven expression of AR target genes, this review will discuss our work in revealing the relative importance of MED1 in various cell line models of prostate cancer.

\section{Distinct Transcriptional Outcomes of Sustained AR Activity in CRPC}

A prominent mechanism by which androgen/AR contributes to ADPC growth and proliferation is through regulation of G1/S-phase cell cycle transit. Reviewed previously, androgen starvation of prostate cancer cells results in G1 arrest as expression levels of cyclin D1 (CCND1) and p21 are diminished, active cyclin/cyclin-dependent kinase (CDK) complex formation is inhibited, and retinoblastoma (RB) tumor suppressor activity is enhanced [47]. We demonstrated in an early study from our lab that AR supports the androgenindependent progression of CRPC cells through G2/M phase of the cell cycle as a primary means of enhancing CRPC proliferation in the absence of hormone [48]. We characterized the gene expression profiles of the ADPC cell line model LNCaP and its derivative CRPC model, abl, over a time course of androgen stimulation and after silencing of AR to find that the basal AR-mediated gene expression profile of CRPC cells differs remarkably from the hormone-stimulated gene expression profile of ADPC cells. This result suggested that continued AR function in CRPC is directed towards the definition of a unique gene expression profile. In fact, ADPCspecific DHT-upregulated genes were enriched within the biological process, "cellular lipid metabolism," while CRPCspecific basal AR-upregulated genes were enriched in "mitotic cell cycle." A more recent comprehensive characterization of a core set of androgen-stimulated, direct AR target genes also revealed metabolic pathways under the control of this signaling axis in two separate cell line models of ADPC: LNCaP (characterized by AR LBD mutation) and VCaP (characterized by AR overexpression) [49]. That metabolic process deregulation, intuitively required to support rapid proliferation of cancer cell populations, appears in independent studies to be the result of AR activity across distinct molecular subtypes of ADPC suggests a therapeutic focus on these downstream products of androgen signaling may be broadly applicable to the initial treatment of prostate cancer.

AR chromatin immunoprecipitation combined with tiled oligonucleotide microarray (ChIP-on-chip) analysis of 
LNCaP and abl cells provided information regarding the genome-wide redistribution of AR binding sites from ADPC to CRPC [48]. Increased enrichment of AR binding within enhancers of cell cycle, and specifically $M$ phase, genes $(C D K 1, C D C 20$, and $U B E 2 C)$ in abl cells indicated a direct mechanism of AR-mediated CRPC cell cycle progression distinct from the androgen-stimulated G1/S phase progression of ADPC cells. It was further demonstrated that epigenetic definition of abl-specific enhancers, marked by histone $\mathrm{H} 3$ lysine 4 mono- and dimethylation ( $\mathrm{H} 3 \mathrm{~K} 4 \mathrm{mel} / 2)$, provided a cell type-specific chromatin environment amenable to androgen-independent, pioneer factor (FoxAl)-mediated AR recruitment to the UBE2C locus. Expression of UBE2C, a ubiquitin-conjugating enzyme involved in mitotic cell cycle progression by promoting the accumulation and activation of the anaphase-promoting complex/cyclosome (APC/C) [50, 51], was finally shown to be strongly correlated with clinical cases of CRPC versus ADPC and necessary for androgenindependent CRPC but not ADPC cell proliferation. A 2012 study further clarified this point by demonstrating that functional-LBD-deficient AR splice variants (e.g., AR-V7), which accumulate in CRPC cells following enzalutamide treatment, promote a gene expression profile that includes $U B E 2 C$ and is largely unique from the full-length AR-mediated gene profile [52]. Importantly, in CRPC cells treated with enzalutamide and in CRPC xenografts treated with abiraterone, expression of AR splice variants, but not full-length AR, was correlated with UBE2C expression, and this relationship was further reflected in the analysis of AR, AR-V7, and UBE2C expression in CRPC tissue microarrays.

In light of the high prevalence of prostate cancer progression following therapy targeting the androgen/AR signaling axis and the overwhelming evidence supporting the sustained, disease-driving activity of AR in the presence of such treatments, it is desirable to continue to identify direct targets of this transcription factor that contribute to AR-mediated cancer cell proliferation. Our study suggests that these targets may be disease state specific and encourages further characterization of unique AR-transcriptional targets across a broad spectrum of prostate cancer severity, which may offer opportunities to address unique drivers of late stage disease. A recent investigation from the Brown lab, describing a role of enhancer of zeste homolog 2 (EZH2) as a coactivator of ARdriven gene expression in CRPC cells, revealed that enhancer cooccupancy of AR and EZH2 facilitates the expression of DNA-repair proteins KIAA0101 and RAD51C [53, 54]. As FoxAl pioneer factor activity was found to support AR-driven G2/M phase gene expression, these combined results indicate that interactions with specific factors may allow AR to drive the expression of genes within nonoverlapping biological processes, adding complexity to its role in supporting CRPC growth. In light of this finding and the recent analysis of AR genomic distribution in CRPC clinical samples, the results of which suggest that CRPC-specific AR binding sites may occur alongside context-specific collaborating transcription factors (e.g., MYC, E2F, and STATs) [55], targeting CRPC-specific $\mathrm{AR}$ collaborators/coactivators could prove a viable means of inhibiting significant portions of AR-mediated transcription profiles in treatment-resistant contexts.
We have explored the therapeutic approach of targeting downstream products of $\mathrm{AR}$ transcriptional activation that contribute specifically to the growth of CRPC by investigating the use of cell-cycle inhibitor 779 (CCI-779), targeting mTOR activity [56]. We found that by downregulating the expressions of CCND1 and UBE2C, CCI-779 treatment resulted in G1 and G2/M phase accumulation, respectively, of two distinct CRPC cells lines (abl and C4-2B), as well as significantly reducing CRPC cell proliferation. These effects were reproducible in an abl xenograft model, where CCI-779 treatment resulted in a $75 \%$ reduction in average tumor volume relative to control and dramatically reduced UBE2C and CCND1 protein expression compared to control tumor tissue. Importantly, as UBE2C expression has been correlated with metastasis in nonprostatic cancers [57-59], CCI-779 treatment or UBE2C knockdown were both found to inhibit CRPC cell invasion potential. Additionally, CCI-779 treatment was able to inhibit the enhanced invasiveness of CRPC cells transiently overexpressing UBE2C. Mechanistically, decreased UBE2C expression was determined to be the result of UBE2C mRNA destabilization and failed coactivator/transcription complex assembly at UBE2C enhancers (involving SRC1/3, p300, MED1 [discussed below], and Pol II), though AR binding was unaffected.

Other lines of evidence suggest that a therapeutic focus on mTOR signaling may be effective in inhibiting AR targetmediated prostate cancer cell proliferation before and after the development of therapeutic resistance. A 2011 study found that expression of the L-type amino acid transporter LAT3 is controlled directly by AR in the presence of androgen while LAT1 expression, controlled by ATF4, was induced upon androgen deprivation [60]. Concordantly, LAT3 overexpression was observed in clinical samples of ADPC compared to benign tissue and samples of CRPC, while LAT1 expression correlated with progression to CRPC. Both transporters maintain intracellular amino acid homeostasis required for mTORC1 activity [61]. A subsequent investigation from the same group found that silencing of LAT family members or inhibiting their activity with the leucine analog $\mathrm{BCH}$ resulted in lost expression of not only UBE2C but also CDK1 and CDC20 in vitro and in xenograft models of CRPC [62]. Thus, addressing critical downstream targets of AR activity may be accomplished via therapeutic strategies focused beyond the canonical androgen/AR signaling axis resulting in potent cell cycle inhibition.

\section{FoxA1: Master Cell Cycle Regulator}

Considerable interest in collaborating factors that contribute to AR transcriptional activity has evolved from analyses of genome-wide AR binding sites in prostate cancer cells, which revealed a number of cooccurring DNA motifs within ARoccupied regions. Chief among the activities that recognize these motifs are the pioneer factors, FoxA1, and GATA2 (discussed below), whose role in determining nuclear receptor (NR, e.g., AR and estrogen receptor [ER]) binding patterns has long been appreciated, as reviewed previously [63]. In short, pioneer factors are understood to engage regions of compact chromatin, facilitating their decondensation in 
advance of ligand-stimulated NR binding. FoxA1 accomplishes this by displacing the linker histone $\mathrm{Hl}$, owing to the structural similarity of its DNA-binding domain to H1 [64-66]. As briefly mentioned above, enrichment of the epigenetic marks $\mathrm{H} 3 \mathrm{~K} 4 \mathrm{mel} / 2$, known to partially determine FoxAl chromatin occupancy [67], within CRPC cell-specific UBE2C enhancers facilitates androgen-independent pioneer factor binding within these gene regulatory elements. The net result is androgen-independent, FoxA1-mediated AR binding at the UBE2C locus and enhanced expression of this clinically relevant oncogene, suggesting that an important role of FoxA1 in CRPC is the reprogramming of AR activity to drive G2/M transit, rather than G1/S cell cycle progression as in ADPC [48].

In a subsequent study, our lab further investigated the observation that, in contrast to FoxA1 silencing in G2/M phase-synchronized CRPC cells resulting in G2/M accumulation, unsynchronized cells exhibited significant G1 arrest following FoxA1 knockdown, suggesting that this pioneer factor may have functional significance throughout the cell cycle [68]. mRNA and protein expression analysis of several G1 phase genes identified CCNE2 and CCNA2 as being robustly overexpressed in CRPC compared to ADPC cells in an androgen-independent yet FoxA1-dependent manner. Silencing of CCNE2 and CCNA2 significantly decreased CRPC cell proliferation in the absence of androgen, and, remarkably, overexpression of either factor in LNCaP cells enhanced their sensitivity and ability to grow in the presence of subphysiological androgen concentrations.

As in our AR study [48], FoxA1 ChIP-on-chip analyses in $\mathrm{LNCaP}$ and abl cells were utilized to provide a picture of genome-wide FoxA1 binding patterns and evidence of its transcriptional control over these G1 phase genes. In general, CRPC cell-specific FoxA1 binding was observed within the vicinity of genes differentially regulated (upregulated and, to a lesser extent, downregulated) in clinical samples of CRPC versus ADPC [69-71]. Increased FoxA1 binding in abl cells was observed at enhancer elements within the CCNE2 locus, corresponding to enhanced coactivator/transcription complex assembly (Pol II, CREB binding protein [CBP], and MED1) and chromatin accessibility in CRPC cells, suggesting direct regulation of CCNE2 by FoxA1. On the other hand, our work determined that CCNA2 upregulation was the result of enhanced E2F1 binding to the CCNA2 promoter subsequent to direct regulation of E2F1 in CRPC cells by enhanced FoxA1 occupancy of E2F1 regulatory elements. Importantly, motif analysis of CRPC-specific FoxA1 binding sites revealed a significant cooccurrence with CREB and MYB recognition motifs in this cellular context. We then demonstrated that MYBL2/FoxA1 and CREB1/FoxA1 cooccupancy of CCNE2 and E2F1 regulatory elements occurs in a codependent fashion and that silencing of either CREB1 or MYBL2 resulted in reduced enhancer chromatin accessibility, androgen-independent expression of CCNE2, CCNA2, and E2F1 in CRPC cells, and CRPC cell proliferation. We finally showed that enhanced CREB1/MYBL2 occupancy of FoxA1bound $U B E 2 C$ and $C D K 1$ regulatory elements was necessary for the androgen-independent overexpression of these G2/M phase genes in abl versus LNCaP cells.
Together these results identified CREB1/MYBL2 as potential FoxAl collaborators facilitating diverse, diseaserelevant transcriptional outcomes in a CRPC context. Positive correlation between MYBL2/CREB1 expression and disease progression from ADPC to CRPC has been reported and may provide some basis for the observed redistribution of FoxA1 to regions cooccupied by these factors in a model of late stage disease $[72,73]$. CREB1 activity has been the focus of several studies in prostate cancer, and ongoing research should focus on elucidating its global impact on FoxAl binding patterns and gene expression profiles. Looking back to our AR study, these results also suggest that FoxAl engages in distinct collaborative relationships as it determines the expression of genes responsible for progression through multiple phases of the cell cycle. Previous studies have shown that AR expression and transactivation potential are significantly reduced in cells immediately following mitosis, providing some rationale for its selective involvement in FoxA1-mediated cell cycle transit during $\mathrm{G} 2 / \mathrm{M}$ phase alone $[74,75]$. It will therefore be important to comprehensively identify collaborators common to FoxA1 transcriptional complexes throughout the cell cycle (e.g., CREB1 and MYBL2) that may serve as therapeutic targets in the inhibition of this master cell cycle regulator.

\section{GATA2 Promotes AR Target Gene Expression}

Though the nature of FoxAl pioneer factor function has been the subject of numerous studies, an in-depth mechanism by which GATA2 contributes to NR chromatin loading remained somewhat elusive prior to a very recent investigation from our lab [76]. Analysis of AR, GATA2, and FoxA1 chromatin immunoprecipitation combined with highthroughput sequencing (ChIP-seq) datasets revealed that approximately $55 \%$ of all androgen-stimulated AR binding sites in LNCaP cells overlapped with basal pioneer factoroccupied regions, and so we asked how GATA2 contributes to $\mathrm{AR}$ genome-wide binding and androgen-responsive gene expression in both ADPC and CRPC cells. We first characterized two novel GATA2 binding sites in the AR gene locus, finding that pioneer factor occupancy at these locations positively regulated the expression of $A R$ itself. We next showed that androgen-independent GATA2 and FoxA1 occupancy of AR target gene loci ( $A B C C 4$ and $A D P G K$ ) occurs in advance of hormone-induced AR binding. AR loading and target gene expression were inhibited by silencing of either pioneer factor, in some cases to levels achieved by silencing of AR. Importantly, we showed that in occupying gene regulatory elements as a prerequisite to AR binding, GATA2 and FoxA1 exhibit site-specific cobinding characteristics (i.e., codependent and independent).

In regions where GATA2 occupancy was independent of FoxA1, silencing of GATA2 but not FoxA1 was sufficient to inhibit androgen-independent recruitment of the histone acetyltransferase (HAT) p300 [77], accumulation of the active epigenetic mark histone $\mathrm{H} 3$ lysine 27 acetylation (H3K27ac) [78], and chromatin accessibility. This result provided a second layer to the role of GATA2 as a pioneer factor in activating chromatin within androgen-responsive gene 
loci, priming them for AR binding and hormone-inducible expression. The central importance of these two GATA2 activities to global AR-DNA binding was demonstrated in the results of AR ChIP-seq assays performed following GATA2 silencing. Here we showed that nearly $80 \%$ of AR binding sites were lost following GATA2 knockdown, and of the 12,529 sites that remained, dramatically reduced ChIP-seq signal intensity was observed in GATA2-silenced conditions. Finally, as it is understood that AR primarily occupies distal enhancer elements within target gene loci, a critical component to AR-mediated gene expression involves chromatin looping from distal regulatory regions to target gene promoters via protein scaffolding facilitated in part by the Mediator coregulatory complex subunit MED1 (discussed in-depth below). We showed that enhanced locus looping between the AR/GATA2/FoxA1-bound ABCC4 enhancer and promoter follows androgen stimulation and that silencing of either GATA2 or FoxA1 resulted in lost MED1 recruitment and inhibited basal chromatin loop formation. Thus, GATA2 plays a third role in supporting AR activity by preforming functional chromatin loops at androgen-responsive gene loci in advance of AR binding.

These results were largely consistent with previous studies of related GATA family pioneer factors in relation to NR activity and clarifying to previous correlative studies suggesting such roles for GATA2 [79-81]. However, to observe an almost exclusively positive relationship of GATA2 with AR in determining its global binding is in contrast to similar studies of FoxA1 in relation to AR $[82,83]$ and of GATA3 in relation to ER [84]. In these investigations, large numbers of new AR and ER binding sites were observed following FoxA1 and GATA3 silencing, respectively, while only 131 new, lowaffinity AR binding sites were found in our analysis [76]. Due to the unique and highly dependent nature of AR activity on GATA2 function, it is of interest to further characterize the impact of GATA2 inhibition in both treatment sensitive and resistant contexts, as expression of this pioneer factor has been positively correlated with more advanced disease [85], and on global gene expression profiles as a foundation for developing novel GATA2-targeted therapeutics for prostate cancer. The Knudsen group demonstrated the feasibility of such a treatment strategy, using the natural compound curcumin to inhibit accumulation of GATA2/p300/histone acetylation at AR target gene loci in cell line models of ADPC and CRPC, leading to reduced target gene expression, in vitro cell proliferation, and xenograft tumorigenesis [79]. This promising approach may offer the added benefit of sparing patients the unwanted side effects of androgen ablation by focusing on a separate determinant of AR activity. As we have demonstrated for FoxA1 [68], the large number of GATA2 binding sites outside AR-bound regions suggests a potential role for GATA2 beyond the androgen/AR signaling axis, and thus additional GATA2 collaborators and downstream targets must be identified and explored.

\section{CRPC-Specific MED1 Activation Drives Chromatin Looping}

We have demonstrated the importance of chromatin looping between AR-bound enhancers and the promoters of AR target genes in both ADPC and CRPC cells in driving gene expression contributing to disease progression $[48,76]$. Up to this point, chromatin loop formation has been discussed in the context of AR-associated coactivator/transcription complexes consisting of pioneer factors, histone modifying enzymes, and the Mediator complex. To address the determinants of locus looping in the absence of AR was of particular interest in light of CRPC heterogeneity characterized by both AR-positive $(\mathrm{AR}+)$ and AR-negative $(\mathrm{AR}-)$ phenotypes [86, 87]. We therefore investigated the factors contributing to enhanced UBE2C expression in the AR- CRPC cell line PC-3 compared to the AR+ LNCaP cell line [88]. In the PC-3 cellular context, $U B E 2 C$ expression was necessary for androgenindependent cell proliferation and G2/M phase transit, similar to previous results in abl cells [48]. We then characterized several cell type-specific UBE2C enhancers exhibiting robust looping to the $U B E 2 C$ promoter and stronger occupancy of H3K4me1/2, FoxA1, MED1, MED17 (another Mediator complex subunit) [89], Pol II, and TATA-box binding protein (TBP) in PC-3 versus LNCaP cells. Importantly, silencing of either FoxA1 or MED1 resulted in decreased chromatin loop formation and $U B E 2 C$ expression in PC-3 cells. It was further shown that PI3 K/Akt-mediated MED1 phosphorylation (pMED1) supports UBE2C expression and locus looping, as pMED1 ChIP and serial ChIP (reChIP) assays revealed higher pMED1 binding to $U B E 2 C$ regulatory elements in PC-3 versus LNCaP cells and direct interactions between enhancer-associated FoxA1/pMED1 complexes and promoter-associated Pol II/TBP transcription complexes. Expressing phosphomutant MED1 in PC-3 cells resulted in a marked reduction in coactivator/transcription complex assembly at the $U B E 2 C$ locus corresponding to decreased locus looping, UBE2C expression, and AR- CRPC cell proliferation. These results were reproducible only in the AR+ CRPC cell line abl but not the AR+ ADPC cell line LNCaP, suggesting that MED1/pMED1 activity is of central importance to CRPC (AR-/+) alone.

The findings identified a potential therapeutic target for CRPC in MED1, a critical determinant of CRPC-specific disease-relevant gene expression. By revealing an important role of PI3 K/Akt in the phosphorylation/activation of MED1, our work also provides a rationale for focusing therapeutic development efforts on this kinase signaling pathway. Phosphatase and tensin homolog (PTEN) loss, resulting in deregulated PI3 K/Akt activity, is a common genetic abnormality of CRPC cases [90, 91], further underscoring the need for future investigations of this treatment strategy. A recent paper from the Fondell group demonstrated that MED1 phosphorylation is mediated by both ERK and Akt, leading to its activation as well as its accumulation [92]. Gene expression analysis of MED1-overexpressing LNCaP cells further revealed that enhanced MED1 activity results not only in mitotic cell cycle progression via expression of $U B E 2 C$, but also in the enhanced expression of antiapoptotic and proinflammatory gene sets, providing evidence of the broad impact of active MED1 signaling on disease-relevant transcription programs. Thus, targeting of multiple kinase pathways could precipitate regression of advanced prostate cancer, acting partially through inhibition of MED1-mediated gene expression. 


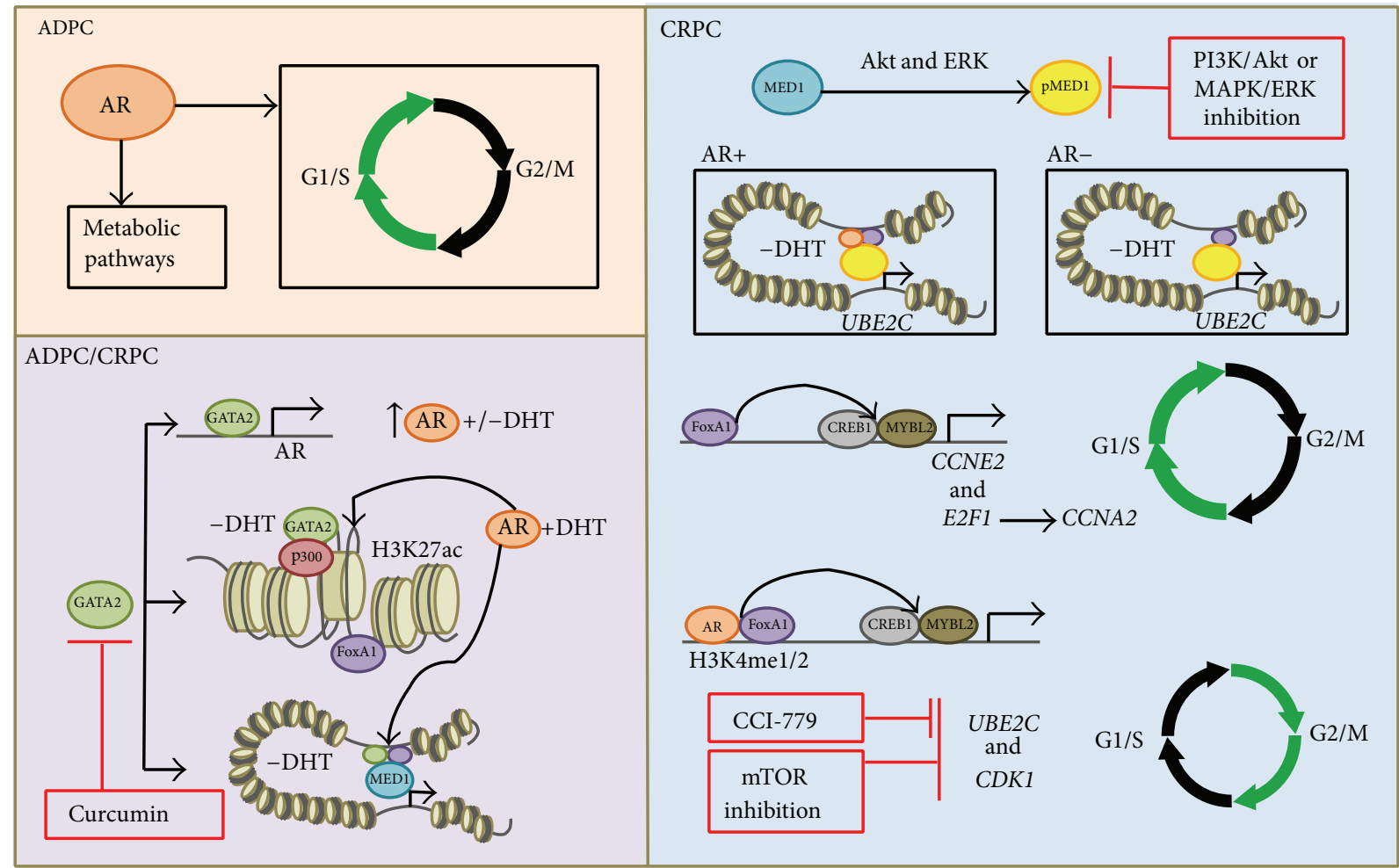

FIGURE 1: Summary of relevant work. In ADPC, androgen-stimulated AR activity drives gene expression profiles related to metabolism and androgen/AR regulates G1/S phase cell cycle progression. In both ADPC and CRPC, the pioneer factor GATA2 contributes to androgenresponsive gene expression via three mechanisms of action: regulating AR expression, promoting activation and accessibility of AR target gene regulatory elements, and aiding in the formation/maintenance of basal chromatin loops between AR target genes enhancers and promoters. In CRPC, Akt and ERK activity leads to MED1 phosphorylation/activation resulting in enhanced locus looping and expression of $U B E 2 C$ in $\mathrm{AR}+$ and AR- cell contexts. Finally, FoxA1 serves as a master cell cycle regulator in CRPC by facilitating AR-mediated expression of G2/M phase cell cycle genes and through a non-AR-associated role in driving G1/S phase progression.

\section{Conclusions and Outlook}

To briefly summarize (Figure 1), the efforts of our lab have contributed to a basic understanding of AR activity, supported by a host collaborating factors and contributing uniquely to various stages of prostate cancer progression [48]. In doing so, we have provided evidence to encourage the continued characterization of AR-mediated gene expression profiles throughout disease states in an effort to identify common as well as disease stage-specific drug targets acting downstream of this transcription factor. We have identified additional activities beyond AR with which the pioneer factor FoxA1 collaborates in determining the expression of both G2/M and G1/S phase cell cycle genes [68], encouraging the search for collaborating factors that may serve as drug targets to inhibit both AR-associated and non-AR-associated FoxA1 function. In determining the most basic means by which GATA2 contributes to AR genome-wide binding in prostate cancer [76], we have revealed a profound dependence of androgen/AR signaling on this pioneer factor, which has been the focus of previous investigations of novel therapeutic strategies in prostate cancer [79]. By providing evidence of the indispensible role of MED1 in both AR+ and AR- CRPC expression of the clinically relevant cell cycle gene UBE2C [88], we have introduced this factor as a potential therapeutic target for disparate molecular subtypes of advanced prostate cancer. Finally, our efforts have provided rationale for the development of novel prostate cancer therapeutics that target PI3 K/Akt signaling upstream of MED1 and mTOR signaling linked to both androgen-dependent and -independent AR activity and contributing to the expression of critical cell cycle genes [56].

\section{Conflict of Interests}

The authors declare that there is no conflict of interests regarding the publication of this paper.

\section{Acknowledgments}

Studies performed in the Laboratory of Qianben Wang were supported by Grants from National Institute of Health (R01 CA151979, U54 CA113001), Department of Defense (W81XWH-12-1-0615), and 2011 V Foundation V Scholar Award, and the Ohio State University Comprehensive Cancer Center. The authors apologize to the colleagues whose relevant work was not cited due to space limitation. 


\section{References}

[1] P. J. Fuller, "The steroid receptor superfamily: mechanisms of diversity," The FASEB Journal, vol. 5, no. 15, pp. 3092-3099, 1991.

[2] K. de Gendt and G. Verhoeven, "Tissue- and cell-specific functions of the androgen receptor revealed through conditional knockout models in mice," Molecular and Cellular Endocrinology, vol. 352, no. 1-2, pp. 13-25, 2012.

[3] M. M. Centenera, J. M. Harris, W. D. Tilley, and L. M. Butler, "The contribution of different androgen receptor domains to receptor dimerization and signaling," Molecular Endocrinology, vol. 22, no. 11, pp. 2373-2382, 2008.

[4] F. Claessens, S. Denayer, N. van Tilborgh, S. Kerkhofs, C. Helsen, and A. Haelens, "Diverse roles of androgen receptor (AR) domains in AR-mediated signaling," Nuclear Receptor Signaling, vol. 6, article e008, 2008.

[5] C. A. Heinlein and C. Chang, "Androgen receptor in prostate cancer," Endocrine Reviews, vol. 25, no. 2, pp. 276-308, 2004.

[6] C. Huggins and P. J. Clark, "Quantitative studies of prostatic secretion: II. The effect of castration and of estrogen injection on the normal and on the hyperplastic prostate glands of dogs," The Journal of Experimental Medicine, vol. 72, no. 6, pp. 747-762, 1940.

[7] C. Huggins and R. A. Stevens, "The effect of castration on benign hypertrophy of the prostate in man," The Journal of Urology, vol. 43, article 105, 1940.

[8] C. Huggins and C. V. Hodges, "Studies on prostate cancer: I. The effects of castration of estrogen and androgen injection on serum phosphatases in metastatic carcinoma of the prostate," Cancer Research, vol. 1, article 203, 1941.

[9] S. R. Denmeade and J. T. Isaacs, "A history of prostate cancer treatment," Nature Reviews Cancer, vol. 2, no. 5, pp. 389-396, 2002.

[10] G. Tolis, D. Ackman, and A. Stellos, "Tumor growth inhibition in patients with prostatic carcinoma treated with luteinizing hormone-releasing hormone agonists," Proceedings of the National Academy of Sciences of the United States of America, vol. 79, no. 5, pp. 1658-1662, 1982.

[11] E. J. Sanford, J. R. Drago, and T. J. Rohner Jr., "Aminoglutethimide medical adrenalectomy for advanced prostatic carcinoma," The Journal of Urology, vol. 115, no. 2, pp. 170-174, 1976.

[12] K. M. Anderson and S. Liao, "Selective retention of dihydrotestosterone by prostatic nuclei," Nature, vol. 219, no. 5151, pp. 277-279, 1968

[13] N. Bruchovsky and J. D. Wilson, "The intranuclear binding of testosterone and 5-alpha-androstan-17-beta-ol-3-one by rat prostate," The Journal of Biological Chemistry, vol. 243, no. 22, pp. 5953-5960, 1968.

[14] W. I. Mainwaring, "A soluble androgen receptor in the cytoplasm of rat prostate," Journal of Endocrinology, vol. 45, no. 4, pp. 531-541, 1969.

[15] S. Liao, D. K. Howell, and T. M. Chang, "Action of a nonsteroidal antiandrogen, flutamide, on the receptor binding and nuclear retention of $5 \alpha$ - dihydrotestosterone in rat ventral prostate," Endocrinology, vol. 94, no. 4, pp. 1205-1209, 1974.

[16] K. E. Knudsen and H. I. Scher, "Starving the addiction: new opportunities for durable suppression of AR signaling in prostate cancer," Clinical Cancer Research, vol. 15, no. 15, pp. 47924798, 2009.

[17] Y. Chen, C. L. Sawyers, and H. I. Scher, "Targeting the androgen receptor pathway in prostate cancer," Current Opinion in Pharmacology, vol. 8, no. 4, pp. 440-448, 2008.
[18] D. A. Loblaw, K. S. Virgo, R. Nam et al., "Initial hormonal management of androgen-sensitive metastatic, recurrent, or progressive prostate cancer: 2006 update of an American Society of Clinical Oncology Practice Guideline," Journal of Clinical Oncology, vol. 25, no. 12, pp. 1596-1605, 2007.

[19] J. D. Debes and D. J. Tindall, "Mechanisms of androgenrefractory prostate cancer," The New England Journal of Medicine, vol. 351, no. 15, pp. 1488-1490, 2004.

[20] B. J. Feldman and D. Feldman, "The development of androgenindependent prostate cancer," Nature Reviews Cancer, vol. 1, no. 1, pp. 34-45, 2001.

[21] K. E. Knudsen and T. M. Penning, "Partners in crime: deregulation of AR activity and androgen synthesis in prostate cancer," Trends in Endocrinology \& Metabolism, vol. 21, no. 5, pp. 315$324,2010$.

[22] C. Tran, S. Ouk, N. J. Clegg et al., "Development of a secondgeneration antiandrogen for treatment of advanced prostate cancer," Science, vol. 324, no. 5928, pp. 787-790, 2009.

[23] H. I. Scher, K. Fizazi, F. Saad et al., "Increased survival with enzalutamide in prostate cancer after chemotherapy," The New England Journal of Medicine, vol. 367, no. 13, pp. 1187-1197, 2012.

[24] G. Attard, A. H. Reid, T. A. Yap et al., "Phase I clinical trial of a selective inhibitor of CYP17, abiraterone acetate, confirms that castration-resistant prostate cancer commonly remains hormone driven," Journal of Clinical Oncology, vol. 26, no. 28, pp. 4563-4571, 2008.

[25] G. Attard, A. H. Reid, R. A'Hern et al., "Selective inhibition of CYP17 with abiraterone acetate is highly active in the treatment of castration-resistant prostate cancer," Journal of Clinical Oncology, vol. 27, no. 23, pp. 3742-3748, 2009.

[26] J. S. de Bono, C. J. Logothetis, A. Molina et al., "Abiraterone and increased survival in metastatic prostate cancer," The New England Journal of Medicine, vol. 364, no. 21, pp. 1995-2005, 2011.

[27] V. Tzelepi, J. Zhang, J.-F. Lu et al., "Modeling a lethal prostate cancer variant with small-cell carcinoma features," Clinical Cancer Research, vol. 18, no. 3, pp. 666-677, 2012.

[28] G. Attard, C. S. Cooper, and J. S. de Bono, "Steroid hormone receptors in prostate cancer: a hard habit to break?" Cancer Cell, vol. 16, no. 6, pp. 458-462, 2009.

[29] M. J. Linja, K. J. Savinainen, O. R. Saramaki, T. L. J. Tammela, R. L. Vessella, and T. Visakorpi, "Amplification and overexpression of androgen receptor gene in hormone-refractory prostate cancer," Cancer Research, vol. 61, no. 9, pp. 3550-3555, 2001.

[30] A. Latil, I. Bieche, D. Vidaud et al., "Evaluation of androgen, estrogen (ER $\alpha$ and $E R \beta)$, and progesterone receptor expression in human prostate cancer by real-time quantitative reverse transcription-polymerase chain reaction assays," Cancer Research, vol. 61, no. 5, pp. 1919-1926, 2001.

[31] T. Hara, J.-I. Miyazaki, H. Araki et al., "Novel mutations of androgen receptor: a possible mechanism of bicalutamide withdrawal syndrome," Cancer Research, vol. 63, no. 1, pp. 149-153, 2003.

[32] M. Korpal, J. M. Korn, X. Gao et al., "An F876L mutation in androgen receptor confers genetic and phenotypic resistance to MDV3100 (enzalutamide)," Cancer Discovery, vol. 3, no. 9, pp. 1030-1043, 2013.

[33] J. D. Joseph, N. Lu, J. Qian et al., "A clinically relevant androgen receptor mutation confers resistance to second-generation antiandrogens enzalutamide and ARN-509," Cancer Discovery, vol. 3, no. 9, pp. 1020-1029, 2013. 
[34] S. M. Dehm, L. J. Schmidt, H. V. Heemers, R. L. Vessella, and D. J. Tindall, "Splicing of a novel androgen receptor exon generates a constitutively active androgen receptor that mediates prostate cancer therapy resistance," Cancer Research, vol. 68, no. 13, pp. 5469-5477, 2008.

[35] R. Hu, T. A. Dunn, S. Wei et al., "Ligand-independent androgen receptor variants derived from splicing of cryptic exons signify hormone-refractory prostate cancer," Cancer Research, vol. 69, no. 1, pp. 16-22, 2009.

[36] Z. Guo, X. Yang, F. Sun et al., "A novel androgen receptor splice variant is up-regulated during prostate cancer progression and promotes androgen depletion-resistant growth," Cancer Research, vol. 69, no. 6, pp. 2305-2313, 2009.

[37] J. A. Locke, E. S. Guns, A. A. Lubik et al., "Androgen Levels increase by intratumoral de novo steroidogenesis during progression of castration-resistant prostate cancer," Cancer Research, vol. 68, no. 15, pp. 6407-6415, 2008.

[38] R. B. Montgomery, E. A. Mostaghel, R. Vessella et al., "Maintenance of intratumoral androgens in metastatic prostate cancer: a mechanism for castration-resistant tumor growth," Cancer Research, vol. 68, no. 11, pp. 4447-4454, 2008.

[39] M. Stanbrough, G. J. Bubley, K. Ross et al., "Increased expression of genes converting adrenal androgens to testosterone in androgen-independent prostate cancer," Cancer Research, vol. 66, no. 5, pp. 2815-2825, 2006.

[40] L. A. Ponguta, C. W. Gregory, F. S. French, and E. M. Wilson, "Site-specific androgen receptor serine phosphorylation linked to epidermal growth factor-dependent growth of castrationrecurrent prostate cancer," The Journal of Biological Chemistry, vol. 283, no. 30, pp. 20989-21001, 2008.

[41] M.-L. Zhu and N. Kyprianou, "Androgen receptor and growth factor signaling cross-talk in prostate cancer cells," EndocrineRelated Cancer, vol. 15, no. 4, pp. 841-849, 2008.

[42] R. Chmelar, G. Buchanan, E. F. Need, W. Tilley, and N. M. Greenberg, "Androgen receptor coregulators and their involvement in the development and progression of prostate cancer," International Journal of Cancer, vol. 120, no. 4, pp. 719-733, 2007.

[43] C. W. Gregory, B. He, R. T. Johnson et al., "A mechanism for androgen receptor-mediated prostate cancer recurrence after androgen deprivation therapy," Cancer Research, vol. 61, no. 11, pp. 4315-4319, 2001.

[44] I. U. Agoulnik, A. Vaid, W. E. Bingman III et al., "Role of SRC1 in the promotion of prostate cancer cell growth and tumor progression," Cancer Research, vol. 65, no. 17, pp. 7959-7967, 2005.

[45] M. P. Steinkamp, O. A. O’Mahony, M. Brogley et al., “Treatmentdependent androgen receptor mutations in prostate cancer exploit multiple mechanisms to evade therapy," Cancer Research, vol. 69, no. 10, pp. 4434-4442, 2009.

[46] American Cancer Society, Cancer Facts and Figures 2012, Atlanta, Ga, USA, American Cancer Society edition, 2012.

[47] S. P. Balk and K. E. Knudsen, "AR, the cell cycle, and prostate cancer," Nuclear Receptor Signaling, vol. 6, article e001, 2008.

[48] Q. Wang, W. Li, Y. Zhang et al., "Androgen receptor regulates a distinct transcription program in androgen-independent prostate cancer," Cell, vol. 138, no. 2, pp. 245-256, 2009.

[49] C. E. Massie, A. Lynch, A. Ramos-Montoya et al., "The androgen receptor fuels prostate cancer by regulating central metabolism and biosynthesis," The EMBO Journal, vol. 30, no. 13, pp. 27192733, 2011.
[50] Y. Ye and M. Rape, "Building ubiquitin chains: E2 enzymes at work," Nature Reviews Molecular Cell Biology, vol. 10, no. 11, pp. 755-764, 2009.

[51] J. H. van Ree, K. B. Jeganathan, L. Malureanu, and J. M. van Deursen, "Overexpression of the E2 ubiquitin-conjugating enzyme UbcH10 causes chromosome missegregation and tumor formation," The Journal of Cell Biology, vol. 188, no. 1, pp. 83-100, 2010.

[52] R. Hu, C. Lu, E. A. Mostaghel et al., "Distinct transcriptional programs mediated by the ligand-dependent full-length androgen receptor and its splice variants in castration-resistant prostate cancer," Cancer Research, vol. 72, no. 14, pp. 3457-3462, 2012.

[53] K. Xu, Z. J. Wu, A. C. Groner et al., "EZH2 oncogenic activity in castration-resistant prostate cancer cells is Polycombindependent," Science, vol. 338, no. 6113, pp. 1465-1469, 2012.

[54] L. K. Povlsen, P. Beli, S. A. Wagner et al., "Systems-wide analysis of ubiquitylation dynamics reveals a key role for PAF15 ubiquitylation in DNA-damage bypass," Nature Cell Biology, vol. 14, no. 10, pp. 1089-1098, 2012.

[55] N. L. Sharma, C. E. Massie, A. Ramos-Montoya et al., "The androgen receptor induces a distinct transcriptional program in castration-resistant prostate cancer in man," Cancer Cell, vol. 23, no. 1, pp. 35-47, 2013.

[56] H. Wang, C. Zhang, A. Rorick et al., "CCI-779 inhibits cellcycle G2-M progression and invasion of castration-resistant prostate cancer via attenuation of UBE2C transcription and mRNA stability," Cancer Research, vol. 71, no. 14, pp. 4866-4876, 2011.

[57] S. Chen, Y. Chen, C. Hu, H. Jing, Y. Cao, and X. Liu, "Association of clinicopathological features with UbcH10 expression in colorectal cancer," Journal of Cancer Research and Clinical Oncology, vol. 136, no. 3, pp. 419-426, 2010.

[58] D. Loussouarn, L. Campion, F. Leclair et al., "Validation of UBE2C protein as a prognostic marker in node-positive breast cancer," British Journal of Cancer, vol. 101, no. 1, pp. 166-173, 2009.

[59] I. W. Cunha, K. C. Carvalho, W. K. Martins et al., "Identification of genes associated with local aggressiveness and metastatic behavior in soft tissue tumors," Translational Oncology, vol. 3, no. 1, pp. 23-32, 2010.

[60] Q. Wang, C. G. Bailey, C. Ng et al., "Androgen receptor and nutrient signaling pathways coordinate the demand for increased amino acid transport during prostate cancer progression," Cancer Research, vol. 71, no. 24, pp. 7525-7536, 2011.

[61] S. R. Kimball, L. M. Shantz, R. L. Horetsky, and L. S. Jefferson, "Leucine regulates translation of specific mRNAs in L6 myoblasts through mTOR-mediated changes in availability of eIF4E and phosphorylation of ribosomal protein S6," The Journal of Biological Chemistry, vol. 274, no. 17, pp. 11647-11652, 1999.

[62] Q. Wang, J. Tiffen, C. G. Bailey et al., "Targeting amino acid transport in metastatic castration-resistant prostate cancer: effects on cell cycle, cell growth, and tumor development," Journal of the National Cancer Institute, vol. 105, no. 19, pp. 14631473, 2013.

[63] K. S. Zaret and J. S. Carroll, "Pioneer transcription factors: establishing competence for gene expression," Genes \& Development, vol. 25, no. 21, pp. 2227-2241, 2011.

[64] J. R. Friedman and K. H. Kaestner, "The Foxa family of transcription factors in development and metabolism," Cellular and Molecular Life Sciences, vol. 63, no. 19-20, pp. 2317-2328, 2006. 
[65] L. A. Cirillo, C. E. McPherson, P. Bossard et al., "Binding of the winged-helix transcription factor HNF3 to a linker histone site on the nucleosome," The EMBO Journal, vol. 17, no. 1, pp. 244254,1998

[66] L. A. Cirillo, F. R. Lin, I. Cuesta, D. Friedman, M. Jarnik, and K. S. Zaret, "Opening of compacted chromatin by early developmental transcription factors HNF3 (FoxA) and GATA-4," Molecular Cell, vol. 9, no. 2, pp. 279-289, 2002.

[67] M. Lupien, J. Eeckhoute, C. A. Meyer et al., "FoxA1 translates epigenetic signatures into enhancer-driven lineage-specific transcription," Cell, vol. 132, no. 6, pp. 958-970, 2008.

[68] C. Zhang, L. Wang, D. Wu et al., "Definition of a FoxA1 cistrome that is crucial for $\mathrm{G}_{1}$ to $S$-phase cell-cycle transit in castrationresistant prostate cancer," Cancer Research, vol. 71, no. 21, pp. 6738-6748, 2011.

[69] E. LaTulippe, J. Satagopan, A. Smith et al., "Comprehensive gene expression analysis of prostate cancer reveals distinct transcriptional programs associated with metastatic disease," Cancer Research, vol. 62, no. 15, pp. 4499-4506, 2002.

[70] Y. P. Yu, D. Landsittel, L. Jing et al., "Gene expression alterations in prostate cancer predicting tumor aggression and preceding development of malignancy," Journal of Clinical Oncology, vol. 22, no. 14, pp. 2790-2799, 2004.

[71] S. Varambally, J. Yu, B. Laxman et al., "Integrative genomic and proteomic analysis of prostate cancer reveals signatures of metastatic progression," Cancer Cell, vol. 8, no. 5, pp. 393-406, 2005.

[72] A. P. Kumar, S. Bhaskaran, M. Ganapathy et al., "Akt/cAMPresponsive element binding protein/cyclin D1 network: a novel target for prostate cancer inhibition in transgenic adenocarcinoma of mouse prostate model mediated by Nexrutine, a Phellodendron amurense bark extract," Clinical Cancer Research, vol. 13, no. 9, pp. 2784-2794, 2007.

[73] D. Wu, H. E. Zhau, W.-C. Huang et al., "cAMP-responsive element-binding protein regulates vascular endothelial growth factor expression: implication in human prostate cancer bone metastasis," Oncogene, vol. 26, no. 35, pp. 5070-5077, 2007.

[74] E. D. Martinez and M. Danielsen, "Loss of androgen receptor transcriptional activity at the $\mathrm{G}_{1} / \mathrm{S}$ transition," The Journal of Biological Chemistry, vol. 277, no. 33, pp. 29719-29729, 2002.

[75] I. V. Litvinov, D. J. Vander Griend, L. Antony et al., "Androgen receptor as a licensing factor for DNA replication in androgensensitive prostate cancer cells," Proceedings of the National Academy of Sciences of the United States of America, vol. 103, no. 41, pp. 15085-15090, 2006.

[76] D. Wu, B. Sunkel, Z. Chen et al., "Three-tiered role of the pioneer factor GATA2 in promoting androgen-dependent gene expression in prostate cancer," Nucleic Acids Research, vol. 42, no. 6, pp. 3607-3622, 2014.

[77] Q. Jin, L.-R. Yu, L. Wang et al., "Distinct roles of GCN5/PCAFmediated $\mathrm{H} 3 \mathrm{~K} 9 \mathrm{ac}$ and $\mathrm{CBP} / \mathrm{p} 300$-mediated $\mathrm{H} 3 \mathrm{~K} 18 / 27 \mathrm{ac}$ in nuclear receptor transactivation," The EMBO Journal, vol. 30, no. 2, pp. 249-262, 2011.

[78] E. Calo and J. Wysocka, "Modification of enhancer chromatin: what, how, and why?" Molecular Cell, vol. 49, no. 5, pp. 825-837, 2013.

[79] S. Shah, S. Prasad, and K. E. Knudsen, "Targeting pioneering factor and hormone receptor cooperative pathways to suppress tumor progression," Cancer Research, vol. 72, no. 5, pp. 12481259, 2012.

[80] F. Hayakawa, M. Towatari, Y. Ozawa, A. Tomita, M. L. Privalsky, and H. Saito, "Functional regulation of GATA-2 by acetylation," Journal of Leukocyte Biology, vol. 75, no. 3, pp. 529-540, 2004.
[81] C. R. Vakoc, D. L. Letting, N. Gheldof et al., "Proximity among distant regulatory elements at the $\beta$-globin locus requires GATA-1 and FOG-1," Molecular Cell, vol. 17, no. 3, pp. 453-462, 2005.

[82] B. Sahu, M. Laakso, K. Ovaska et al., "Dual role of FoxA1 in androgen receptor binding to chromatin, androgen signalling and prostate cancer," The EMBO Journal, vol. 30, no. 19, pp. 3962-3976, 2011.

[83] D. Wang, I. Garcia-Bassets, C. Benner et al., "Reprogramming transcription by distinct classes of enhancers functionally defined by eRNA," Nature, vol. 474, no. 7351, pp. 390-394, 2011.

[84] V. Theodorou, R. Stark, S. Menon, and J. S. Carroll, "GATA3 acts upstream of FOXA1 in mediating ESR1 binding by shaping enhancer accessibility," Genome Research, vol. 23, no. 1, pp. 1222, 2013.

[85] M. Bohm, W. J. Locke, R. L. Sutherland, J. G. Kench, and S. M. Henshall, "A role for GATA-2 in transition to an aggressive phenotype in prostate cancer through modulation of key androgenregulated genes," Oncogene, vol. 28, no. 43, pp. 3847-3856, 2009.

[86] R. B. Shah, R. Mehra, A. M. Chinnaiyan et al., "Androgenindependent prostate cancer is a heterogeneous group of diseases: lessons from a rapid autopsy program," Cancer Research, vol. 64, no. 24, pp. 9209-9216, 2004.

[87] Z. G. Li, P. Mathew, J. Yang et al., "Androgen receptor-negative human prostate cancer cells induce osteogenesis in mice through FGF9-mediated mechanisms," The Journal of Clinical Investigation, vol. 118, no. 8, pp. 2697-2710, 2008.

[88] Z. Chen, C. Zhang, D. Wu et al., "Phospho-MED1-enhanced UBE2C locus looping drives castration-resistant prostate cancer growth," The EMBO Journal, vol. 30, no. 12, pp. 2405-2419, 2011.

[89] S. Malik and R. G. Roeder, "The metazoan Mediator co-activator complex as an integrative hub for transcriptional regulation," Nature Reviews Genetics, vol. 11, no. 11, pp. 761-772, 2010.

[90] L. Li, M. M. Ittmann, G. Ayala et al., "The emerging role of the PI3-K-Akt pathway in prostate cancer progression," Prostate Cancer and Prostatic Diseases, vol. 8, no. 2, pp. 108-118, 2005.

[91] P. K. Majumder and W. R. Sellers, "Akt-regulated pathways in prostate cancer," Oncogene, vol. 24, no. 50, pp. 7465-7474, 2005.

[92] F. Jin, S. Irshad, W. Yu et al., "ERK and AKT signaling drive MED1 overexpression in prostate cancer in association with elevated proliferation and tumorigenicity," Molecular Cancer Research, vol. 11, no. 7, pp. 736-747, 2013. 


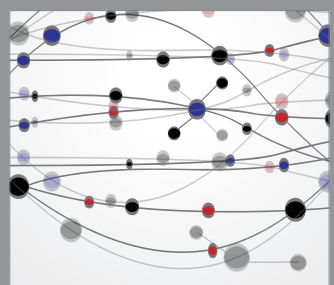

The Scientific World Journal
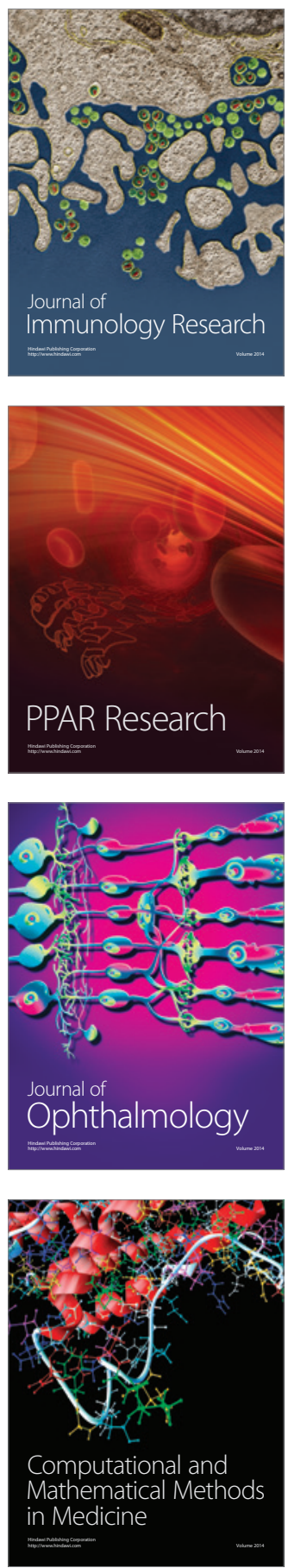

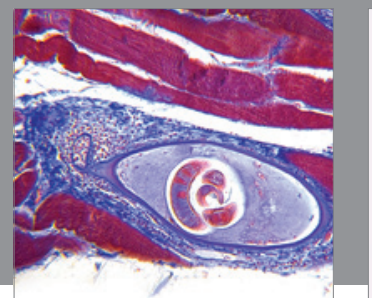

Gastroenterology

Research and Practice
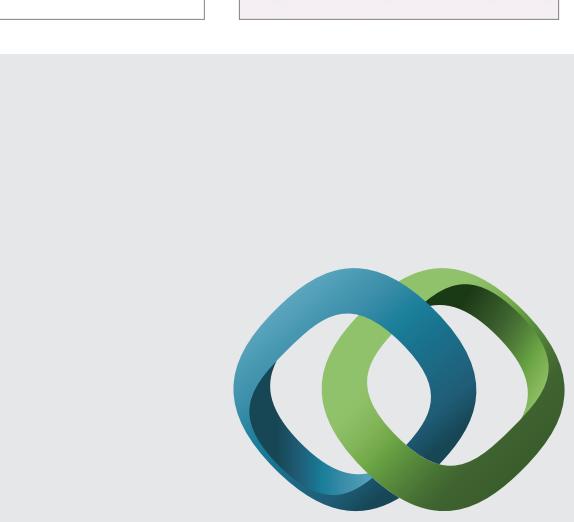

\section{Hindawi}

Submit your manuscripts at

http://www.hindawi.com
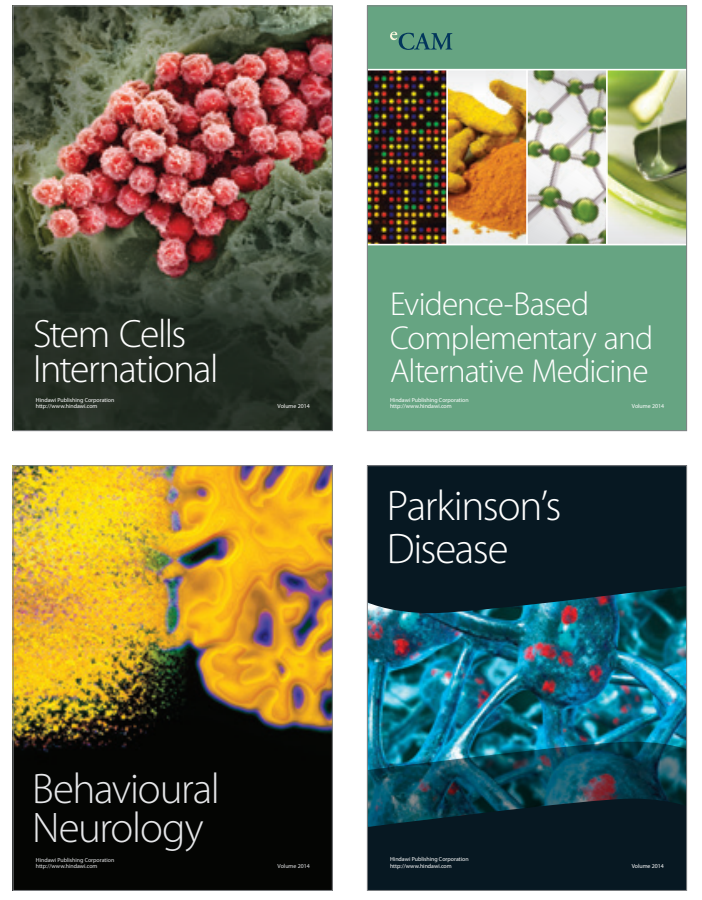
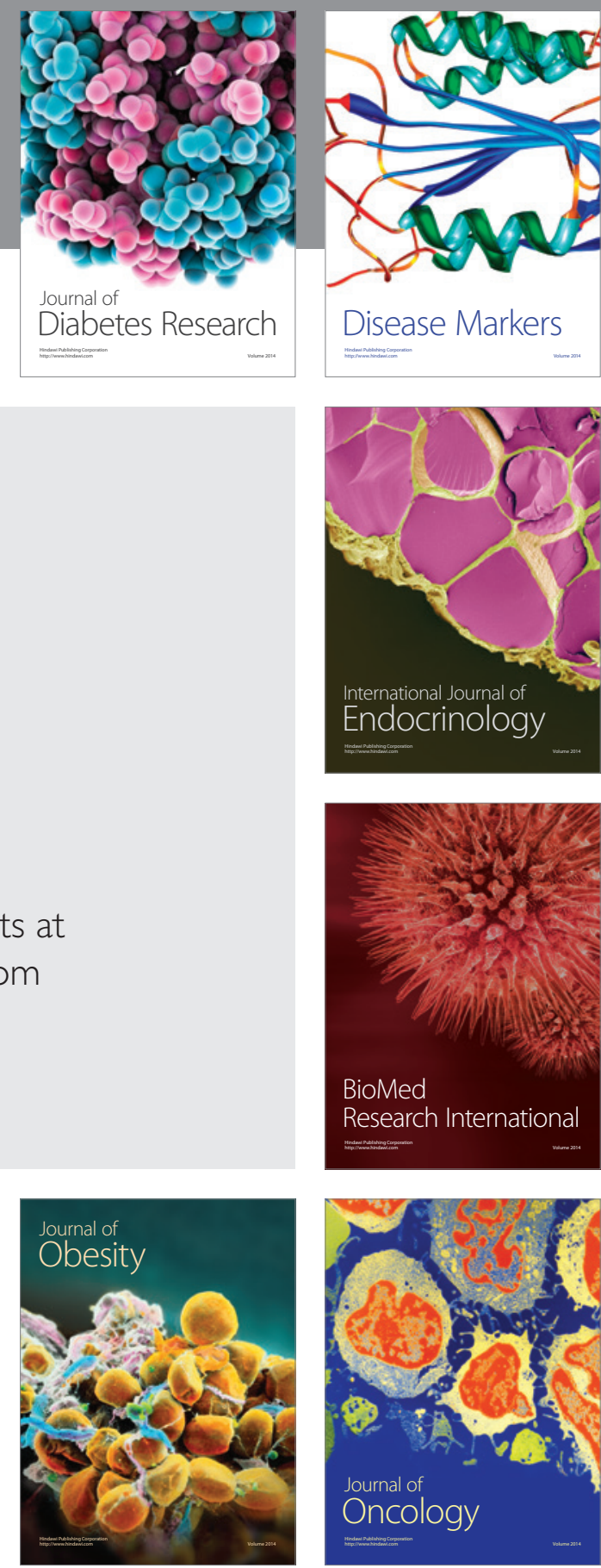

Disease Markers
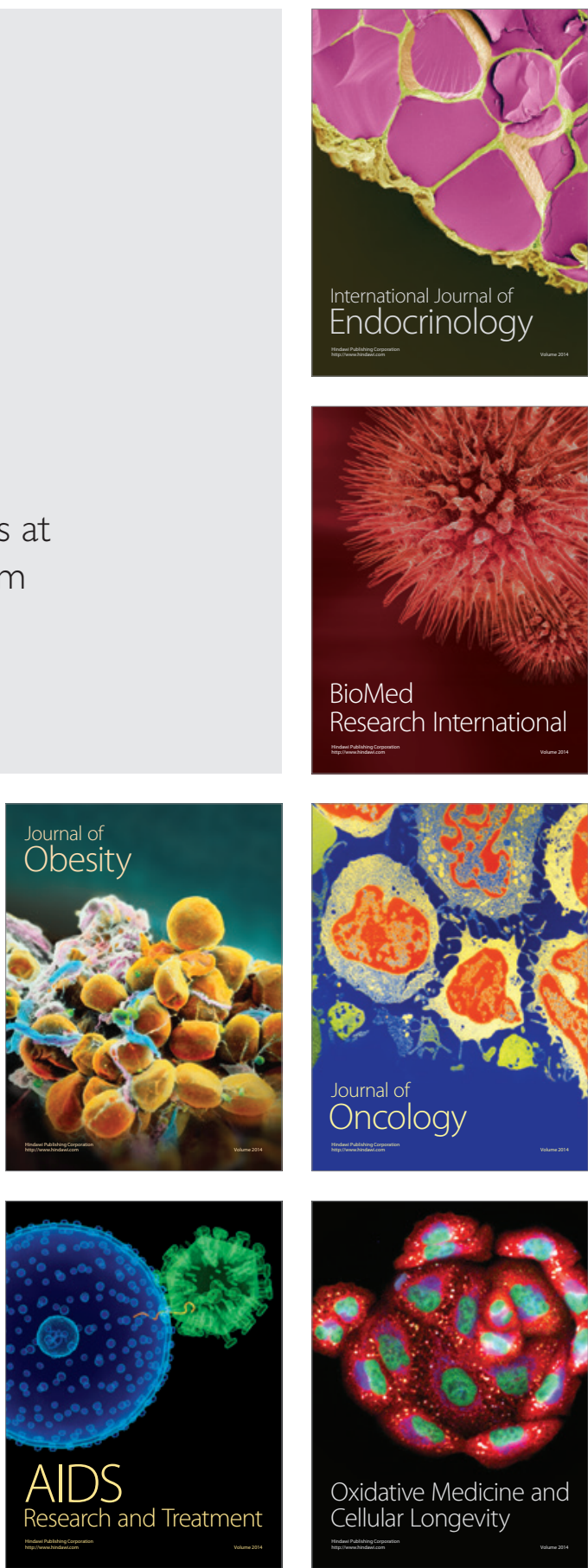